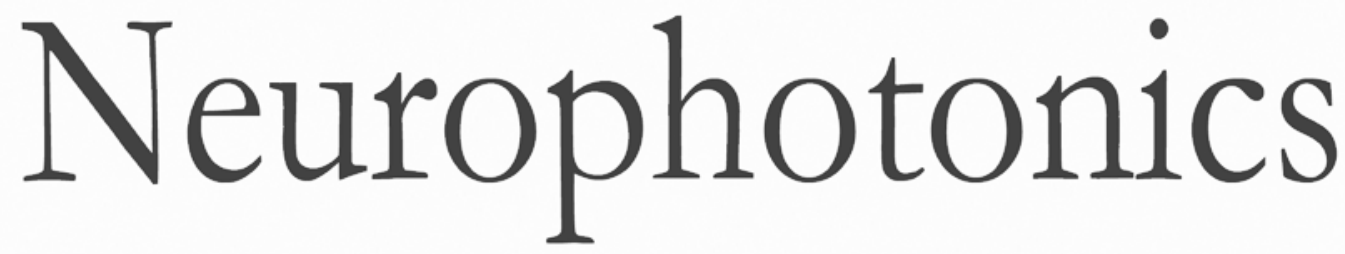

\title{
Speckle reduction in visible-light optical coherence tomography using scan modulation
}

\author{
Ian Rubinoff \\ Lisa Beckmann \\ Yuanbo Wang \\ Amani A. Fawzi \\ Xiaorong Liu \\ Jenna Tauber \\ Katie Jones \\ Hiroshi Ishikawa \\ Joel S. Schuman \\ Roman Kuranov \\ Hao F. Zhang
}




\title{
Speckle reduction in visible-light optical coherence tomography using scan modulation
}

\author{
Ian Rubinoff, ${ }^{a}$ Lisa Beckmann, ${ }^{a}$ Yuanbo Wang, ${ }^{b}$ Amani A. Fawzi, ${ }^{c}$ Xiaorong Liu, ${ }^{\mathrm{d}}$ Jenna Tauber, ${ }^{\mathrm{e}}$ Katie Jones, ${ }^{\mathrm{e}}$ \\ Hiroshi Ishikawa, ${ }^{e}$ Joel S. Schuman, ${ }^{e}$ Roman Kuranov, ${ }^{a, b}$ and Hao F. Zhang ${ }^{a, c, *}$ \\ ${ }^{a}$ Northwestern University, Department of Biomedical Engineering, Evanston, Illinois, United States \\ ${ }^{\mathrm{b}}$ Opticent Health, Evanston, Illinois, United States \\ "Northwestern University, Department of Ophthalmology, Chicago, Illinois, United States \\ dUniversity of Virginia, Department of Biology and Psychology, Charlottesville, Virginia, United States \\ eNew York University, Department of Ophthalmology, New York, United States
}

\begin{abstract}
We present a technique to reduce speckle in visible-light optical coherence tomography (vis-OCT) that preserves fine structural details and is robust against sample motion. Specifically, we locally modulate B-scans orthogonally to their axis of acquisition. Such modulation enables acquisition of uncorrelated speckle patterns from similar anatomical locations, which can be averaged to reduce speckle. To verify the effectiveness of speckle reduction, we performed in-vivo retinal imaging using modulated raster and circular scans in both mice and humans. We compared speckle-reduced vis-OCT images with the images acquired with unmodulated $\mathrm{B}$-scans from the same anatomical locations. We compared contrast-to-noise ratio (CNR) and equivalent number of looks (ENL) to quantify the image quality enhancement. Speckle-reduced images showed up to a 2.35-dB improvement in CNR and up to a 3.1-fold improvement in ENL with more discernable anatomical features using eight modulated A-line averages at a $25-\mathrm{kHz}$ A-line rate. ๑ The Authors. Published by SPIE under a Creative Commons Attribution 4.0 Unported License. Distribution or reproduction of this work in whole or in part requires full attribution of the original publication, including its DOI. [DOI: 10.1117/1.NPh.6.4.041107]

Keywords: optical coherence tomography; visible light; speckle; retina; imaging; clinic.

Paper 19045SSR received May 13, 2019; accepted for publication Aug. 8, 2019; published online Sep. 3, 2019.
\end{abstract}

\section{Introduction}

Optical coherence tomography (OCT) is a scattering-based imaging technology that acquires high-resolution threedimensional images of biological samples in vivo. ${ }^{1}$ Following its initial report in 1991, OCT has become the "gold standard" for noninvasive retinal imaging. Today, it is an essential technology in labs and clinics for studying and managing a wide variety of retinal diseases. ${ }^{2}$ Advances in optoelectronics in the past 25 years has led to improved resolution, signal-to-noise ratio (SNR), and imaging field of view (FOV) in OCT. ${ }^{3}$ However, speckle, an image artifact caused by the self-interference of coherent light at random phases, remains a significant source of reduced image quality. ${ }^{4}$ This is of particular salience in retinal imaging, where speckle noise can obscure fine structures in the outer retina, such as the retinal pigment epithelium (RPE) and Bruch's membrane (BM). Minute pathological changes in these structures may be strongly associated with the progressions of several retinal diseases, including macular degeneration ${ }^{5}$ and central serous retinopathy (CSR). ${ }^{6}$

To improve the imaging quality, researchers have developed several methods to suppress speckle artifacts in OCT. These methods can be classified into two categories: digital filtering ${ }^{7}$ and incoherent averaging. ${ }^{8}$ Digital filtering, while simple to implement and effective in reducing the grainy appearance of speckle, causes blurring that degrades image resolution and prevents the delineation of fine anatomical features. Incoherent averaging, on the other hand, samples photons that have undergone statistically different scattering events, thereby generating

*Address all correspondence to Hao F. Zhang, E-mail: hfzhang@north western.edu uncorrelated speckle patterns. Uncorrelated patterns from similar structural locations can be averaged to remove the speckle and reveal the original anatomical information. The physical basis of incoherent averaging method makes it ideal for situations where the study of fine anatomical features is required. However, manipulating image acquisition to obtain uncorrelated speckle patterns can be challenging. First, different scattering events must be probed without losing the structural integrity of the location of interest. Second, multiple acquisitions at a particular location are required to generate enough patterns suitable for averaging. Samples with strong motion can pose a challenge to averaging, especially in human eyes. Multiple approaches have been developed to achieve incoherent averaging of speckle while retaining high image quality. The most basic technique is to average consecutive B-scans in a raster pattern, either from the same location or from a slightly offset position. ${ }^{9}$ The former relies on a small sample movement to modify scattering events, and the latter directly modifies scattering events across consecutive separated B-scans. More advanced techniques for spatial averaging include modulating the scanning beam after every A-line with a translational offset ${ }^{10}$ or angular offset. ${ }^{11}$ In particular, translational offset has been shown to be more robust against sample motion than B-scan averaging. Both scan modulations have been previously implemented with additional hardware and moving parts. Other techniques include multiwavelength averaging, ${ }^{12,13}$ modulation of light wavefront, ${ }^{14}$ and nonlocal, software-based averaging. ${ }^{15}$

Recent development of visible-light optical coherence tomography (vis-OCT) has generated new capabilities for retinal imaging, including visualization of fine structures with ultrahigh resolution and spectroscopic analysis of blood-oxygen concentration $\left(\mathrm{sO}_{2}\right) .{ }^{16-18}$ Speckle, which distorts both structural and 
spectral information, ${ }^{19}$ can undermine these new benefits. To reduce speckle in vis-OCT using incoherent averaging, there are some unique challenges. First, there is strong optical absorption and scattering in tissue in the visible-light spectral range, reducing the amount of photons that can be collected per unit time. This is coupled with high relative intensity from the supercontinuum laser source. ${ }^{16}$ To achieve high SNR, a prolonged camera exposure is required, therefore reducing scanning speed. Second, since eyes are sensitive to visible-light illumination, vis-OCT often suffers from increased retinal motion. Finally, preservation of structure-dependent spectroscopic information requires anatomically localized sampling of scattering events.

A method for speckle reduction that is both structurally localized and robust against retinal motion is optimal for vis-OCT. Furthermore, it is ideal for a speckle reduction technique to be easily implementable in clinical settings to increase usability, reduce likelihood of malfunction, and cause no additional discomfort to patients. A straightforward approach is to perform B-scan averaging. However, eye motion in vis-OCT makes B-scan averaging unreliable due to blurring, even with postacquisition image registration. To overcome this challenge, we modulated the scanning trajectory orthogonally to the direction of the B-scan images during data acquisition. This method enabled sampling of different speckle patterns while maintaining high anatomical similarity between modulations. We implemented scanning modulation by directly controlling the galvanometer scanners without additional hardware.

\section{Methods}

\subsection{Scanning Protocol}

We modulated both raster and circular scans, which are commonly used in vis-OCT, ${ }^{16}$ to test our speckle reduction method. Figure 1 illustrates the modulated raster scan [Figs. 1(a) and 1(b)] and modulated circular scan [Figs. 1(c) and 1(d)]. As shown in Figs. 1(a) and 1(c), we define the B-scan axis as the direction along which a traditional cross-sectional image would be acquired without modulation. We define the orthogonal axis as the direction orthogonal to the B-scan axis on the twodimensional scanning plane. Movement along the orthogonal axis [arrow 1 in Figs. 1(b) and 1(d)] occurs in $n$ equidistant steps, where $n$ is the number of speckle-uncorrelated A-lines to be locally averaged. Each translation of the galvanometer [red dots in Figs. 1(b) and 1(d)] is discrete, synchronized with the spectrometer camera exposure, and implemented entirely via software control without additional hardware or moving parts. This avoids a complex synchronization procedure or risk of desynchronization between the beam path and the camera exposure when using an external scanner. ${ }^{10}$ The centroids of each spot generating an A-line are separated by a distance $d$ along the orthogonal axis [Fig. 1(b)]. After $n$ translations in this direction, the beam is shifted along the B-scan axis [arrow 2 in Figs. 1(b) and 1(d)], followed by a reversed scan along the orthogonal axis [arrow 3 in Figs. 1(b) and 1(d)]. Such modulation superimposes a rectangular wave on the B-scan axis, where each rising and falling edge of each rectangle contains $n$ speckle-uncorrelated A-lines. While other modulation shapes such as sinusoidal or triangular are possible, we chose rectangular to best preserve lateral resolution along the B-scan axis. During acquisition, several parameters, including $n, d$, and imaging FOV are adjustable. We investigate how to obtain an optimal $d$ in Sec. 3.1.
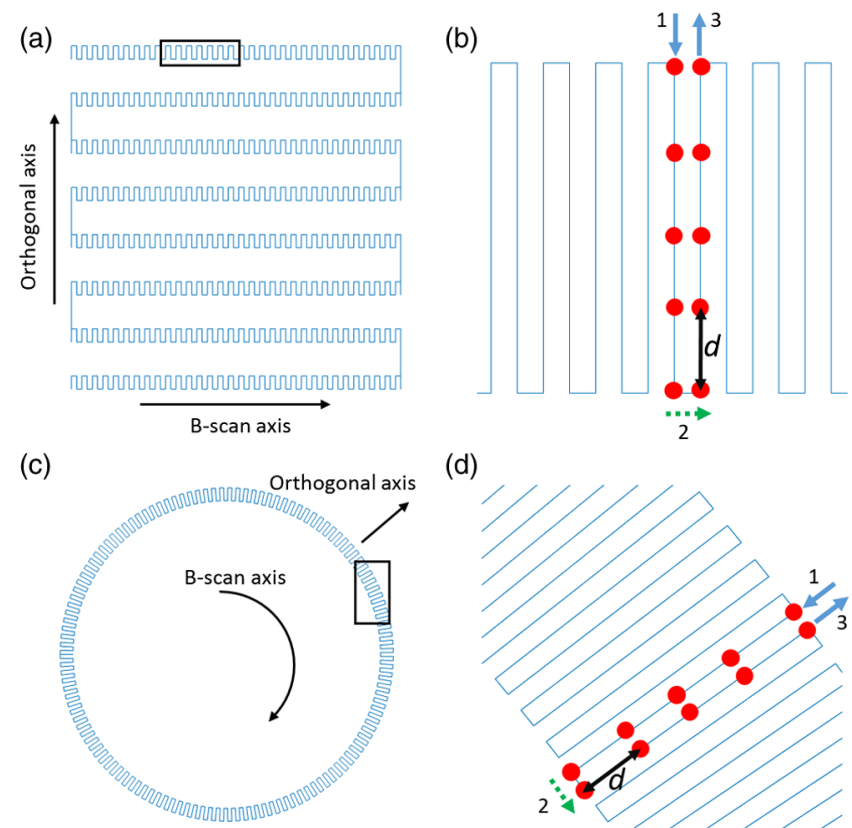

(d)

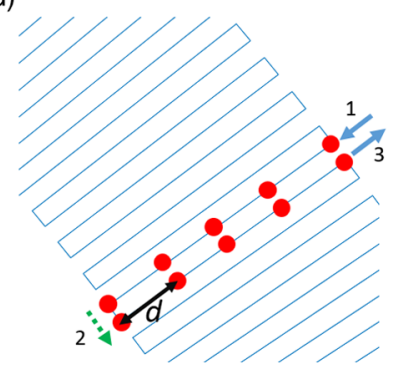

Fig. 1 Illustrations of speckle-reduction scanning protocols. (a) Overall illustration of the relationship between the B-scan axis and the orthogonal axis in the modulated raster scan; (b) detailed illustration of the A-line acquisition sequence in the modulated raster scan. Here, $d$ is the distance between two adjacent A-lines along the orthogonal axis. The arrows 1,2 , and 3 highlight the trajectory of galvanometer motion. (c) Overall illustration of the relationship between the B-scan axis and the orthogonal axis in the modulated circular scan; (d) detailed illustration of the A-line acquisition sequence in the modulated circular scan.

We averaged all $n$ A-lines in the orthogonal direction along each rectangular edge [Figs. 1(b) and 1(d)] to generate a single speckle-reduced A-line (srA-line). For a desired sampling density of $m$ srA-lines per speckle-reduced B-scan (srB-scan), the total number of camera acquisitions per srB-scan is $n \times m$. Each consecutive srA-line in an srB-scan can then be calculated as

$\operatorname{srA}_{j}=\frac{1}{n} \sum_{i=1}^{n} A_{i j} \quad$ for $j=0,1,2, \ldots m-1$,

where $i$ is the index of each set of $n$ A-lines about the edge of a rectangle; $j$ is the index of each consecutive edge of a rectangle; and $A_{i j}$ is the $i+j \times n$ 'th A-line in a full B-scan acquisition.

Since an srB-scan increases imaging time over normal B-scan acquisition by a factor of $n$, it is important to collectively limit $n, m$, and the number of total srB-scans to prevent overly long imaging time. First, all sampling numbers were selected in powers of 2 to support fast graphics processing unit data processing. Next, we limited all imaging experiments to 8192 total A-lines per srB-scan. Given a camera exposure time of $40 \mu \mathrm{s}$, which is required for sufficiently high SNR, an srB-scan could be acquired in $328 \mathrm{~ms}$, an upper limit for reducing bulk motion artifacts (satisfying Nyquist criterion of $500 \mathrm{~ms}$ for eye microsaccades of $\sim 1 \mathrm{~Hz}$ ). ${ }^{20}$ Furthermore, we chose to limit the total image acquisition time to $\sim 5 \mathrm{~s}$ to prevent patient fatigue and discomfort. This limited the total amount of srB-scans per acquisition to 16 (5.25 s total acquisition time). In our experimental human imaging system (Sec. 2.3.2), we maximized lateral sampling density in a raster scan without spot overlap, where $m=1024$ srA-lines and $n=8$ averages. The parameters 
$n, m$, and $d$ can be easily modified for different experimental conditions.

The srB-scan averages along the same locations as $n$ spatially separated B-scans, each of $m$ A-lines. However, in direct B-scan averaging, each A-line at a particular lateral position is delayed temporally by the scanner's fly-back time. This results in a total sampling period of $n \times m \times t$, where $t$ is the camera exposure time for each A-line. In our method, modulation removes the wait for scanner fly-back, thereby reducing the total sampling period to $n \times t$ for each srA-line. Using our experimental parameters ( $m=1024, n=8, t=40 \mu \mathrm{s})$, we reduced the sampling period from $328 \mathrm{~ms}$ to $320 \mu$ s for each srA-line and increased the srA-line rate from 3 to $3125 \mathrm{~Hz}$. Constant, involuntary retinal motions ${ }^{20}$ can occur at frequencies up to $90 \mathrm{~Hz}$ with amplitudes up to 40 arc sec (equivalent to $0.011-\mu \mathrm{m}$ change in sampling location per $40-\mu$ s camera exposure in the human retina). This leaves the possibility of only $0.088 \mu \mathrm{m}$ of movement during an srA-line, which is insignificant when compared with the micron-order lateral and axial resolutions in OCT. Therefore, the improved srA-line rate is highly significant.

\subsection{Metrics to Evaluate Image Quality Improvement}

We used contrast-to-noise ratio (CNR) and equivalent number of looks $(\mathrm{ENL})^{21}$ to evaluate image quality improvement after speckle reduction. CNR measures how well the sample feature can be discerned from the surrounding background. Mean intensity and variance from both the image background and the sample feature are included to account for two separate noise components: intrinsic OCT background noise and speckle. Since the optical properties of different features vary, we calculated the $\mathrm{CNR}(\mathrm{dB})$ in confined region of interests (ROIs) as

$$
\mathrm{CNR}=10 \log _{10} \frac{\mu_{i}-\mu_{b}}{\sqrt{\sigma_{i}^{2}+\sigma_{b}^{2}}},
$$

where $\mu_{i}$ is the mean intensity of the $i$ 'th ROI; $\mu_{b}$ is the mean intensity of the background outside of the sample structure; $\sigma_{i}^{2}$ is the variance of the $i$ 'th ROI; and $\sigma_{b}^{2}$ is the variance of the background outside of the sample feature.

ENL is the squared inverse of the speckle contrast and measures the smoothness and homogeneity within an ROI. We calculated ENL as

$$
\mathrm{ENL}=\frac{\mu_{i}^{2}}{\sigma_{i}^{2}},
$$

where $\mu_{i}$ is the mean intensity of the $i^{\prime}$ th ROI and $\sigma_{i}^{2}$ is the variance of the $i$ 'th ROI. An increase in ENL serves as a strong indicator for the reduction of speckle.

We compared CNR and ENL in srB-scans with a "reference" B-scan from the same location as an srB-scan. A reference B-scan included 8192 A-lines acquired along the B-scan axis with a $40-\mu$ s camera exposure. Every eight consecutive A-lines were averaged, resulting in a final sampling density of 1024 averaged A-lines per reference B-scan. This operation was equivalent to acquiring an srB-scan without modulating the scanner along the orthogonal axis. Because of high sampling density along the B-scan axis, averaged speckle patterns were still highly correlated, preventing reduction of speckle. However, background noise was equally suppressed in reference B-scans and srB-scans because they used the same amount of temporal averaging. We compared the CNR and ENL values between the reference B-scan and srB-scans to evaluate the effectiveness of speckle reduction.

\subsection{Data Acquisition}

We tested our speckle reduction protocol in both mouse and human retinas using two prototype systems developed at Northwestern University. In addition, we further tested our speckle reduction method in humans in a clinical setting using a commercial vis-OCT system (Aurora X1, Opticent Health), where optical engineering expertise was unavailable. We directly implemented the modulated scanning protocol in that system without additional calibration, alignment, or changes to the photographer's workflow.

\subsubsection{Mouse imaging}

For mouse imaging, we used the system described in our previous work. ${ }^{22}$ In brief, a $1 / e^{2}$ spot size of $\sim 5.7 \mu \mathrm{m}$ was incident on the retina. We controlled the total illumination power to $1.2 \mathrm{~mW}$ on the cornea in all instances. For a raster scan, we used $m=1024, n=8$, and $d=6.3 \mu \mathrm{m}$. An FOV of $1.4 \times 1.4 \mathrm{~mm}^{2}$ was used in all mouse retina images, equivalent to $\sim 1.4-\mu \mathrm{m}$ separation between srA-lines along the B-scan axis. For a circular scan, we used $n=8$ and $d=5.4 \mu \mathrm{m}$. The circle circumference was $1.8 \mathrm{~mm}$, equivalent to $\sim 1.8-\mu \mathrm{m}$ separation between srA-lines along the B-scan axis. Improved from our previous system, we adopted a commercial spectrometer (Blizzard SR, Opticent Health) with a 2048-pixel line scan camera covering 510 to $610 \mathrm{~nm}$, which provided an axial resolution of $\sim 1 \mu \mathrm{m}$ in tissue. We used an A-line rate of $25 \mathrm{kHz}$ in all rodent experiments.

All rodent experimental procedures were approved by the Northwestern University IACUC and conformed to the Association for Research in Vision and Ophthalmology (ARVO) Statement on Animal Research. We anesthetized adult C57B16/J mice $(n=8)$ with an intraperitoneal injection $(10 \mathrm{~mL} / \mathrm{kg}$ body weight) of a ketamine/xylazine cocktail (ketamine: $11.45 \mathrm{mg} / \mathrm{mL}$; xylazine: $1.7 \mathrm{mg} / \mathrm{mL}$ ). Each mouse was then placed on a custom-made animal holder and immobilized for imaging. The body temperature was maintained with a heat lamp. To dilate the pupil, we applied a drop of $1 \%$ tropicamide hydrochloride ophthalmic solution. Throughout imaging, we applied one drop of commercial artificial tears after each image acquisition to prevent corneal dehydration. After the imaging session concluded, the mouse was allowed to recover under heat lamp and was returned to the animal housing facility.

\subsubsection{Human imaging}

Human imaging was performed using two vis-OCT systems. First, images were acquired in the Ophthalmology Department at Northwestern Memorial Hospital using an experimental system reported in our previous work. ${ }^{18}$ We controlled the illumination power to be $<250 \mu \mathrm{W}$ on the cornea in all our human studies. A $1 / e^{2}$ spot size of $\sim 6.3 \mu \mathrm{m}$ was incident on the retina. For a raster scan, we used $n=8, d=7 \mu \mathrm{m}$, and $m=1024$. The FOV was $6.8 \times 6.8 \mathrm{~mm}^{2}$, leading to $\sim 6.6-\mu \mathrm{m}$ separation between srA-lines along the B-scan axis. For a circular scan, we used $n=8$ and $d=5.9 \mu \mathrm{m}$. The circle circumference was $18.3 \mathrm{~mm}$, equivalent to $\sim 18-\mu \mathrm{m}$ separation between srA-lines along the B-scan axis. Similar to our mouse system, we 
upgraded to a commercial spectrometer (Blizzard SR, Opticent Health). The A-line rate was $25 \mathrm{kHz}$ in all human imaging tests.

To verify the efficacy of our method outside the lab environment, we further conducted human imaging in the Department of Ophthalmology at New York University (NYU) Langone Medical Center. A clinical vis-OCT system (Aurora X1, Opticent Health) was used to acquire all images. It offered an axial resolution of $\sim 1 \mu \mathrm{m}$ and we controlled the spectrometer exposure time to be $40 \mu \mathrm{s}$. We used the same raster scanning parameters as those in the Northwestern system but reduced the FOV to $5 \times 5 \mathrm{~mm}^{2}$. We implemented the speckle-reduction acquisitions in Aurora X1 entirely via a software update without any additional calibration or hardware modifications. Clinical photographers acquired retinal images without any changes to their normal workflow.

All human imaging procedures in the respective imaging locations were approved by the Northwestern University Institutional Review Board (IRB) and NYU IRB and adhered to the tenets of the Declaration of Helsinki. Healthy volunteers without known eye diseases provided informed consent before imaging (Northwestern site: $n=3$; NYU site: $n=6$ ).

\subsection{Initial Calibration for Orthogonal Spot Separation}

A calibration procedure was needed for coarse determination of optimal spot separation, $d$, along the orthogonal axis. Since CNR is associated with the ability to discern features from noise, we used it as the primary indicator for image quality. In theory, an increased $d$ increases the decorrelation of the speckle patterns between adjacent orthogonal A-lines. After averaging, speckle is maximally reduced when the averaged patterns are entirely uncorrelated. ${ }^{10}$ However, if $d$ is too large, we will lose structural similarity between orthogonal A-lines, which can result in image blurring. We investigated the impact of modulation distance on CNR by imaging a model mouse eye using both raster and circular scans. The model eye was made from a silica bead (diameter: $3.15 \mathrm{~mm}$ ). We attached two layers of tape and paper with an ink pattern to the bottom of the bead to simulate the retinal layers. Using the rodent vis-OCT system, we reached a $1 / e^{2}$ spot size of $\sim 5.5 \mu \mathrm{m}$ on the tape layers through the bead. We then varied the $d$ value from 0 to $13.75 \mu \mathrm{m}$ in 16 steps and acquired an srB-scan after each step. We calculated CNR from three ROIs in the top tape layer and averaged them to determine the impact of the $d$ value on image quality.

\section{Results}

\subsection{Impact of Modulation Distance on Image Quality}

The results to identify an optimal $d$ value are shown in Fig. 2 . Figures 2(a) and 2(b), respectively, show how CNR values vary as a function of $d$ in imaging the model eye using modulated raster and circular scans. When $d$ is increased from 0 to $13.75 \mu \mathrm{m}$ in both scans, CNR reaches its maximum at $d=$ $6 \mu \mathrm{m}$. Figures 2(c) and 2(e) show raster srB-scans with $d=0$ and $6 \mu \mathrm{m}$, respectively. Figures 2(d) and 2(f) show magnified views of the two highlighted images [yellow boxes in Figs. 2(c) and 2(e)], respectively. The srB-scan with $d=6 \mu \mathrm{m}$ [Fig. 2(f)] shows a smoother intensity distribution within each layer and much improved discrimination between the tape and the paper layers, as compared with the unmodulated scan [Fig. 2(d)].
Figures 2(g)-2(j) show the similar comparison in the circular scan, where the speckle-reduced circular scan with $d=6 \mu \mathrm{m}$ demonstrates improvement in image quality.

Figures 2(k) and 2(1), respectively, show the pixel intensity histograms from the tape layer 1 [highlighted in Fig. 2(c)] in the raster and circular scans. In both scan patterns, the intensity histograms changed from a broad, right-skewed distribution when $d=0 \mu \mathrm{m}$ to a lower-variance, nearly centrosymmetric distribution when $d=6 \mu \mathrm{m}$. These results agree with the expected change in pixel intensity distribution from Rayleigh distribution to Poisson distribution after speckle reduction. ${ }^{14}$

As shown in Figs. 2(a) and 2(b), we used a -0.25-dB drop in CNR to determine the range of acceptable $d$ values, which gives $d_{\min }=5.1 \mu \mathrm{m}$ and $d_{\max }=7.8 \mu \mathrm{m}$. This range is helpful for human retinal imaging, where the eye shape, optical properties, and scanning location may differ among subjects. We also noted that the optimal $d=6 \mu \mathrm{m}$ is approximately equal to the estimated spot size of $\sim 5.5 \mu \mathrm{m}$ on the retina. This suggests that adjacent spots along the orthogonal axis should be as close as possible without spatial overlap. This result is consistent with the notion that spatial overlapping provides correlated speckle patterns. This result also suggests that it is acceptable to estimate the optimal $d$ using the OCT focal spot size on the sample. These considerations are not expected to change in the living human eye, where local movement during a single srA-line $(0.088 \mu \mathrm{m})$ is significantly less than $d$.

We adjusted the $d$ value within the identified range in rodent and human imaging to accommodate different eye conditions. Since we control the $d$ value by the galvanometer angle, we identified optimal angular step size along the orthogonal axis in different experimental conditions. For mouse imaging, the optimal angular step sizes were $0.175 \mathrm{deg}$ and $0.15 \mathrm{deg}$, which correspond to $d$ values of 6.3 and $5.4 \mu \mathrm{m}$, respectively, in raster and circular scans. For human imaging, the optimal angular step sizes were $0.025 \mathrm{deg}$ and $0.02 \mathrm{deg}$, which correspond to $d$ values of 7 and $5.9 \mu \mathrm{m}$, respectively, in raster and circular scans.

\subsection{Speckle Reduction in the Mouse Retina}

Figure 3 shows the speckle reduction results in a mouse retina using a raster scan. Figures 3(a) and 3(b) are the reference $\mathrm{B}$-scan and srB-scan images, respectively. The imaged retina in the srB-scan has a smoother, less grainy appearance that provides a clearer differentiation between anatomical layers. We selected six ROIs from the inner plexiform layer [IPL, highlighted by (a1) and (b1)], outer nuclear layer [ONL, highlighted by (a2) and (b2)], and outer retinal layer [ORL, highlighted by (a3) and (b3)] to quantify quality improvement. Figures 3(a1)3(a3) and Figs. 3(b1)-3(b3) show the magnified views of the six selected ROIs and Table 1 shows the quantitative comparisons of CNR and ENL values from these ROIs. Speckle reduction is particularly helpful in the ORL, where a small gap near RPE and $\mathrm{BM}$ layers is revealed [Fig. 3(b3)], which is not visible in the reference B-scan image [Fig. 3(a3)]. The capability to differentiate RPE and BM may add significant value to various preclinical studies using mouse models.

Figure 4 shows the speckle reduction results in a mouse retina using circular scan. Figures 4(a) and 4(b) are the reference B-scan and srB-scan images, respectively. Again, the srB-scan image improved the overall image quality with better differentiated fine anatomical features. We also selected six ROIs from IPL [highlighted by (a1) and (b1)] and retinal blood vessels 
(a)

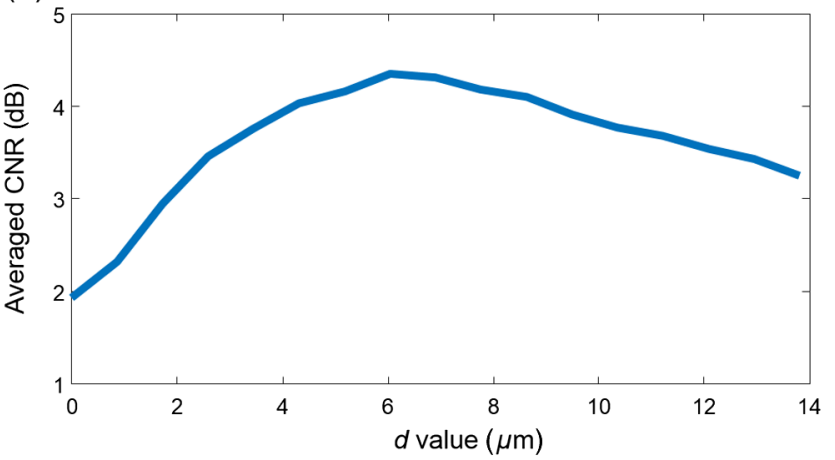

(c)

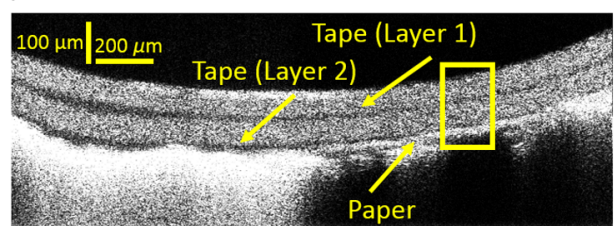

(e)

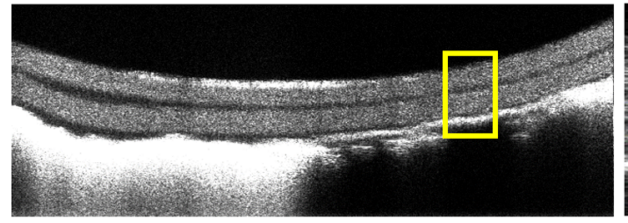

(k)
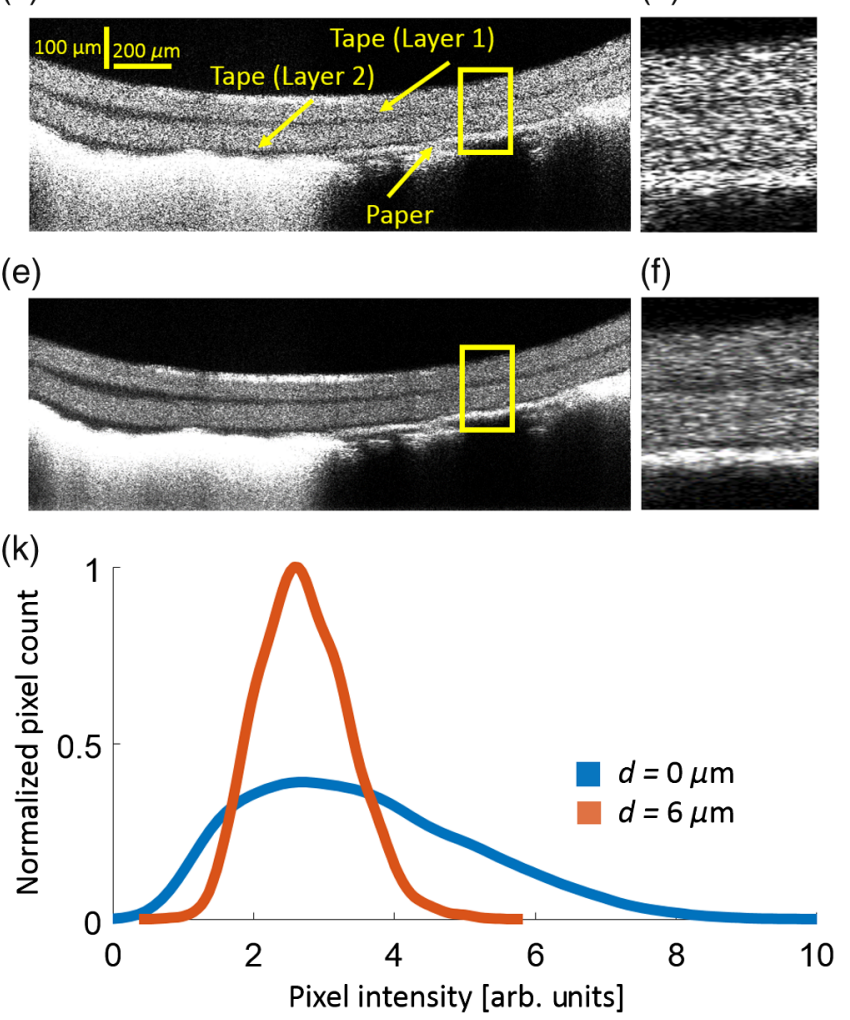

(f)

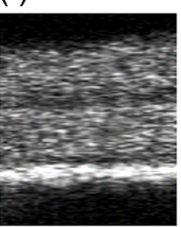

(b)

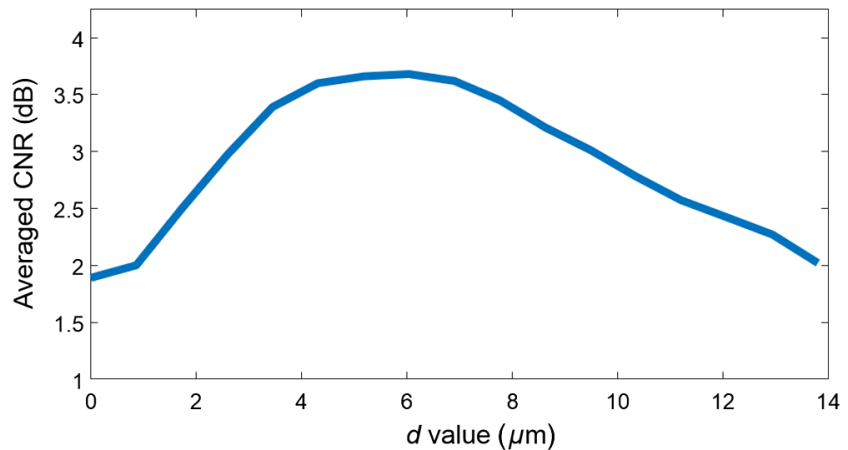

(g)

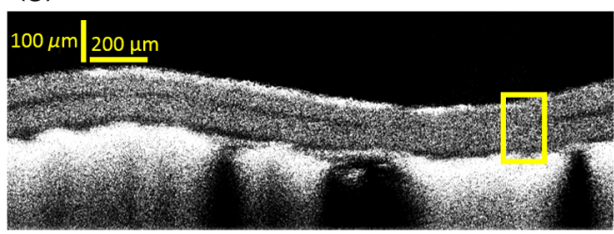

(h)

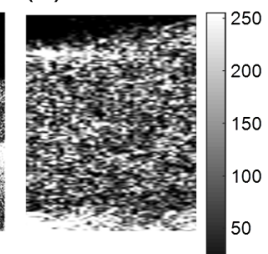

(i)

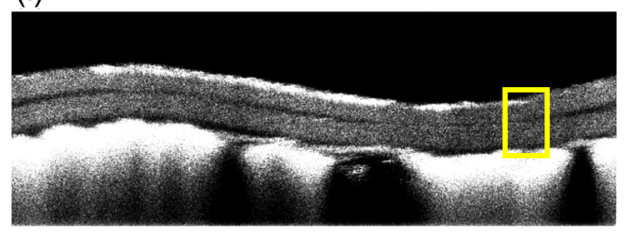

(j)

(l)

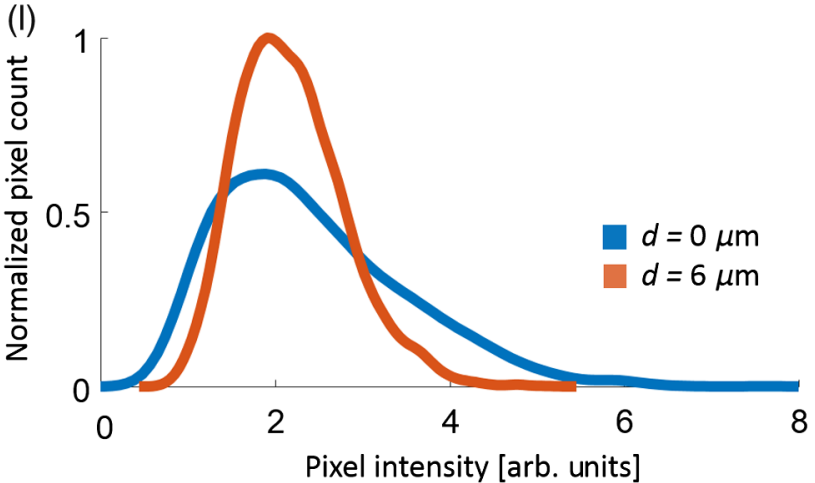

Fig. 2 Speckle-reduction test in the model mouse eye. (a) Change of averaged CNR as a function of $d$ in the modulated raster scan; (b) change of averaged CNR as a function of $d$ in the modulated circular scan. (c) An srB-scan image of the model mouse eye acquired using modulated raster scan with $d=0 \mu \mathrm{m}$. The structures corresponding to the two tape and one paper layers are highlighted by the arrows. (d) Magnified view of the region highlighted by the box in panel (c). (e) An srB-scan image of the model mouse eye acquired using modulated raster scan with $d=6 \mu \mathrm{m}$. (f) Magnified view of the region highlighted by the box in panel (e). (g) An srB-scan image of the model mouse eye acquired using modulated circular scan with $d=0 \mu \mathrm{m}$. (h) Magnified view of the region highlighted by the box in panel $(\mathrm{g})$. (i) An srB-scan image of the model mouse eye acquired using modulated circular scan with $d=6 \mu \mathrm{m}$. (j) Magnified view of the region highlighted by the box in panel (i). All images are plotted with identical color bar. (k) Fitted pixel-intensity histograms within the tape layer 1 acquired by modulated raster scans with $d=0$ and $6 \mu \mathrm{m}$; (I) fitted pixel-intensity histograms within the tape layer 1 acquired by modulated circular scans with $d=0$ and $6 \mu \mathrm{m}$.

[highlighted by (a2), (b2), (a3), and (b3), respectively] for quantitative evaluation.

Table 1 compares the CNR and ENL values from the selected ROIs in both raster and circular scans. In each scan mode, we see increased metric values from the ROIs in the srB-scan images. For raster scans, the ROIs in the IPL, ONL, and ORL show $2.35,1.84$, and $1.32 \mathrm{~dB}$ respective improvements in CNR, and $3.1,2.53$, and 1.84 times respective improvements in ENL.
For circular scans, the ROIs in the IPL and two vessels show $1.84,1.90$, and $1.11 \mathrm{~dB}$ respective improvement in CNR, and $2.69,2.56$, and 1.63 times respective improvements in ENL. CNR and ENL improvements for the ORL in the raster scan and second vessel in the circular scan are slightly lower than other improvements. This is because some of the image background is unavoidably included in the ROI, artificially contributing low pixel intensities to $\mu_{i}$ in the metrics. 
(a)

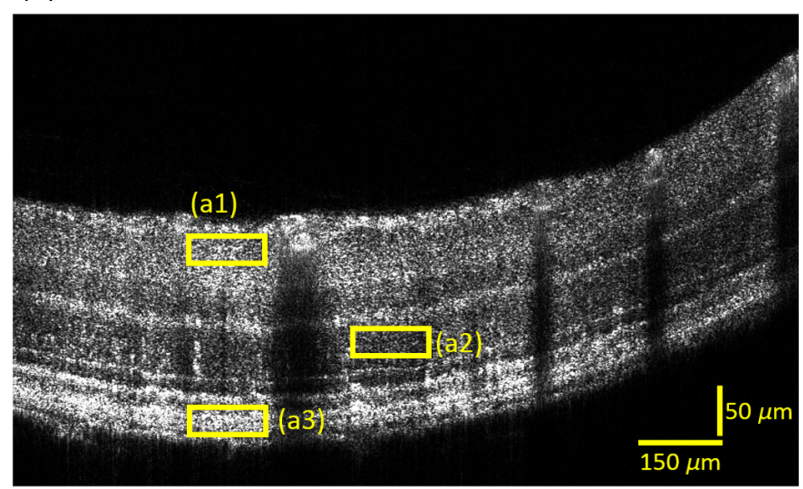

(a1)

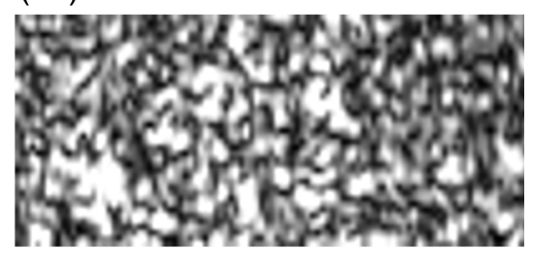

(b1)

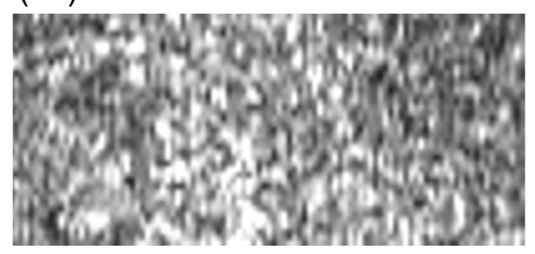

(b2) (b)

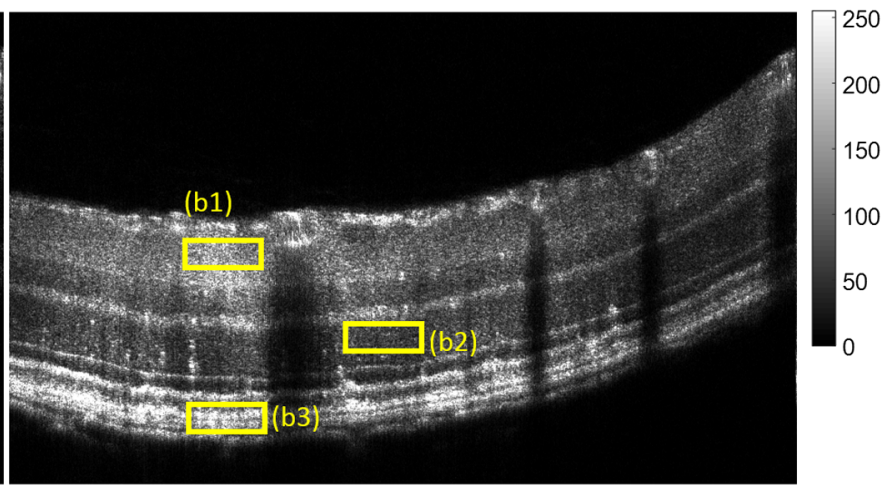

(a3)
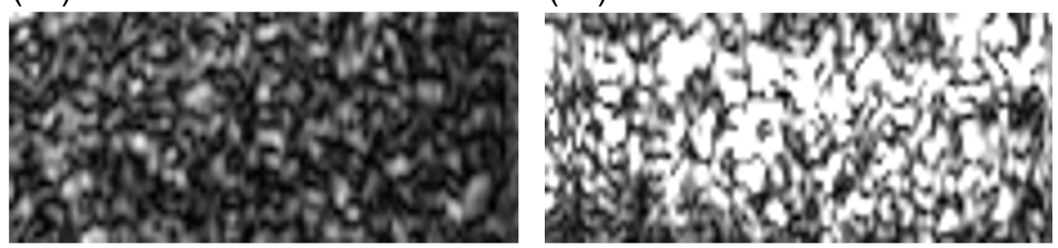

(b3)
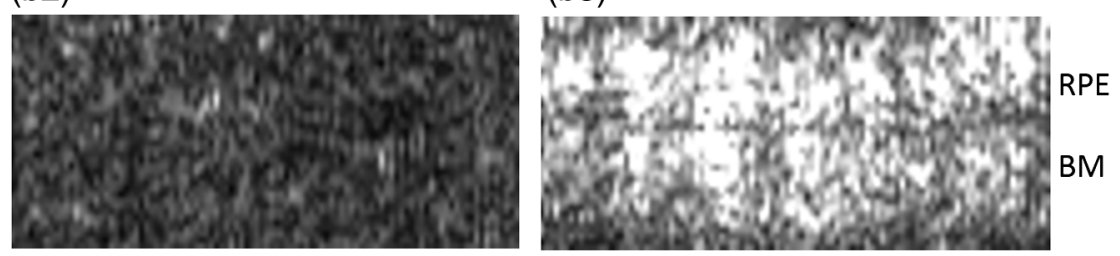

Fig. 3 Speckle-reduction test in mouse retina using modulated raster scan. (a) Reference raster B-scan image. Three ROIs from IPL, ONL, and ORL are highlighted by (a1), (a2), and (a3), respectively. The size of each ROI is $140 \mu \mathrm{m}$ (lateral) $\times 20 \mu \mathrm{m}$ (axial). (b) The corresponding srB-scan image. The same three ROIs are highlighted by (b1), (b2), and (b3), respectively. (a1)-(a3) The magnified views of the three highlighted ROIs in panel (a). (b1)-(b3) The magnified views of the three highlighted ROls in panel (b). CNR and ENL values are calculated from all the selected ROls. All images are plotted with identical color bar.

\subsection{Speckle Reduction in the Human Retina}

We accomplished speckle reduction in the human retina using both the laboratorial prototype $\mathrm{e}^{18}$ and a clinical vis-OCT system. Unlike mouse imaging, in which retinal motion can be minimized and images can be acquired over an extended period, human imaging usually suffers from severe retinal motions and image acquisition needs to complete within few seconds. For visOCT, retinal motion can be much stronger as described in Sec. 1.

Figure 5 shows the speckle reduction results using raster scan in a human retina (22-year-old male volunteer). Figures 5(a) and 5(b) are reference B-scan and srB-scan images superior to the optic disk, respectively. The srB-scan is smoother and less grainy in appearance than the reference B-scan, increasing visibility of the retinal layers. Improved image quality here is consistent with that in the mouse retina [Fig. 3(b)]. We selected six ROIs from the nerve fiber layer [NFL, highlighted by (a1) and (b1)], ganglion cell layer [GCL, highlighted by (a2) and (b2)], and ORLs [highlighted by (a3) and (b3)] to quantify quality improvement. Figures 5(a1)-5(a3) and 5(b1)-5(b3) show the magnified views of the six selected ROIs and Table 2 shows the quantitative comparisons of CNR and ENL values from these ROIs. Of particular note is the increased clarity of ORL in the srB-scan [Fig. 5(b3)]. Unlike the reference B-scan [Fig. 5(a3)], the shape and boundaries of the rod outer segment tips (ROST),
RPE, and BM become clearly discernable from one another. The thickness of $\mathrm{BM}$ is measured as $\sim 3 \mu \mathrm{m}$ and is resolved in the whole image without blur or distortion. The average measured thickness of $\mathrm{BM}$ in the human eye is $\sim 2$ to $5 \mu \mathrm{m},{ }^{23}$ which is consistent with our measurement. The distinct separation between the BM and the RPE, as shown in Fig. 5(a3), may open up new window to investigate macular degeneration, where initial pathological alterations are hypothesized to start from BM. ${ }^{23,24}$ Finally, we note a shadow caused by a small blood vessel as highlighted by the arrows in both the reference B-scan [Fig. 5(a3)] and the srB-scan [Fig. 5(b3)] images in ORL. It is measured as 2 pixels laterally or $\sim 14 \mu \mathrm{m}$ in width. This feature is better resolved in the srB-scan image, indicating that lateral resolution has been well preserved after speckle reduction.

Repetitive B-scan averaging is not trivial due to retinal motion, which often leads to image blurring even after registration. We overcame this challenge and showed that our speckle reduction method is robust against retinal motion in Fig. 6. We acquired eight repeated raster B-scans (each containing 1024 A-lines) from the same anatomical location and volunteer, as shown in Figs. 5(a) and 5(b). All the B-scans were axially and laterally registered using an fast Fourier transform based cross-correlation algorithm. ${ }^{25}$ The averaged B-scan image [Fig. 6(a)] shows blurred anatomical layers in both the inner retina and the outer retina due to motion. Figure 6(b) shows 

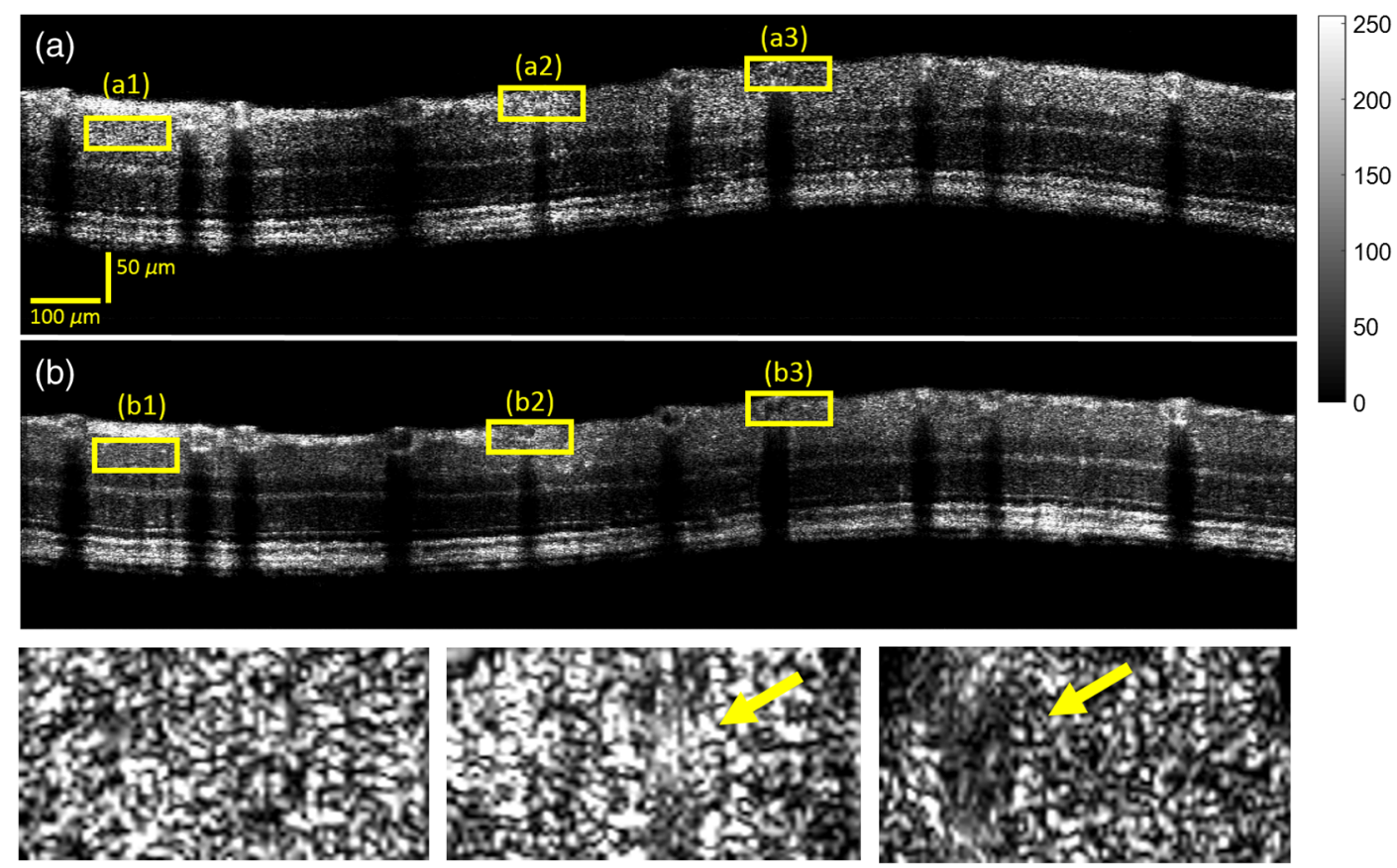

(a1)

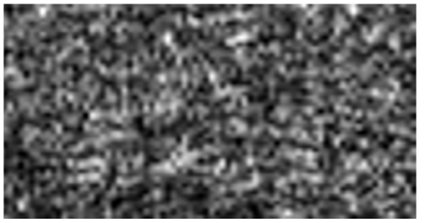

(b1)

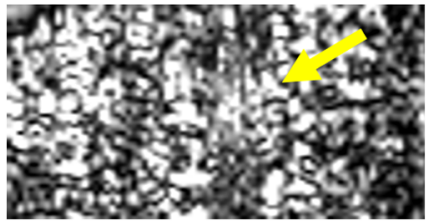

(a2)

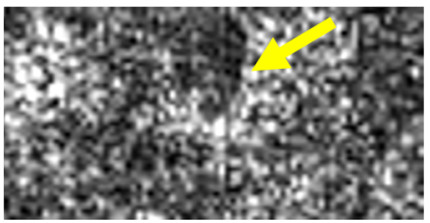

(b2)

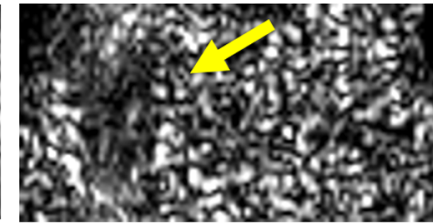

(a3)

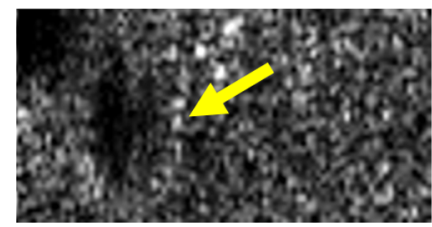

(b3)

Fig. 4 Speckle-reduction test in mouse retina using modulated circular scan. (a) Reference circular Bscan image. Three ROls from IPL and two vessels are highlighted by (a1), (a2), and (a3), respectively. The size of each ROI is $140 \mu \mathrm{m}$ (lateral) $\times 20 \mu \mathrm{m}$ (axial). (b) The corresponding srB-scan image. The same three ROls are highlighted by (b1), (b2), and (b3), respectively. CNR and ENL values are calculated from all the selected ROls. All images are plotted with identical color bar.

Table 1 Image quality metric values from the ROls in the mouse retina shown in Figs. 3 and 4.

\begin{tabular}{lccccc}
\hline Scan type & ROI & $\begin{array}{c}\text { CNR (dB) } \\
\text { ref. B-scan }\end{array}$ & $\begin{array}{c}\text { CNR (dB) } \\
\text { srB-scan }\end{array}$ & $\begin{array}{c}\text { ENL } \\
\text { ref. B-scan }\end{array}$ & $\begin{array}{c}\text { ENL } \\
\text { srB-scan }\end{array}$ \\
\hline Raster & IPL & 2.03 & 4.38 & 3.94 & 12.21 \\
Raster & ONL & 1.63 & 3.47 & 3.38 & 8.56 \\
Raster & ORL & 1.83 & 3.15 & 2.76 & 5.07 \\
Circular & IPL & 2.32 & 4.16 & 4.74 & 12.74 \\
Circular & Vessel 1 & 1.63 & 3.53 & 3.36 & 8.60 \\
Circular & Vessel 2 & 2.00 & 3.11 & 6.11 & 9.97 \\
\hline
\end{tabular}

a magnified view of the region highlighted by the box in Fig. 6(a). Two A-lines from the locations highlighted by lines 1 and 2 in Fig. 6(b) are shown in Fig. 6(c). A-line 1 reveals five anatomical layers in the outer retina, notably with reduced contrast near the RPE. A-line 2 fails to resolve any anatomical features. Since visOCT offers an axial resolution of near $1 \mu \mathrm{m}$, small misalignments in B-scan averaging may lead to much severer blurring. The image quality shown in Fig. 6(a) is representative of most averaged B-scan images acquired by vis-OCT using similar scan parameters. Figure 6(d) shows a magnified view of the same anatomical position from an srB-scan image, where all anatomical layers are clearly resolved across the whole image. The same A-line locations from Fig. 6(b) are highlighted in Fig. 6(d) (by 3 and 4). Figure 6(e) shows A-line 3 and A-line 4, confirming that all ORLs are well resolved despite retinal motion.

We also demonstrate speckle reduction in circular scan in the human retina (Fig. 7). Figures 7(a) and 7(b) show a reference B-scan image and an srB-scan image, acquired at the same anatomical location, respectively. We selected six ROIs from the same locations as in Fig. 5, including the NFL [highlighted by (a1) and (b1)], GCL [highlighted by (a2) and (b2)], and ORL [highlighted by (a3) and (b3)] to quantify quality improvement. Figures 7(a1)-7(a3) and 7(b1)-7(b3) show magnified views of the six selected ROIs and Table 2 shows the quantitative comparisons of the CNR and ENL values from these ROIs. Similar to the ORL in the raster srB-scan [Fig. 6(b3)], the ORL in the circular srB-scan [Fig. 7(b3)] shows distinct separation between BM, RPE, and ROST. In the reference B-scan image [Fig. 7(a3)], however, boundaries of these anatomical layers are not easily differentiated due to speckles. To the best of our knowledge, this is the first demonstration of speckle-reduced imaging in a circular pattern using localized scan modulation in the human retina. 
(a)

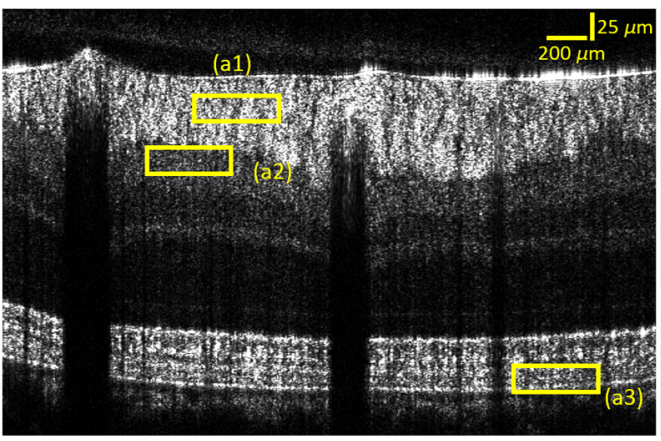

(a1)

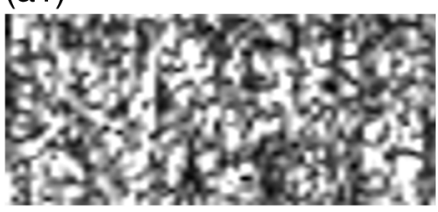

(b1)

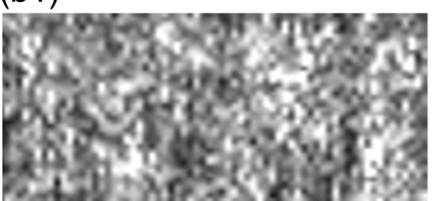

(b2) (b)

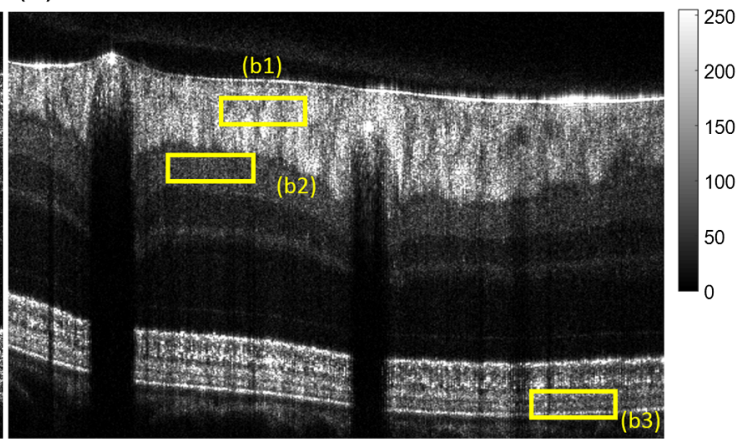

(a3)
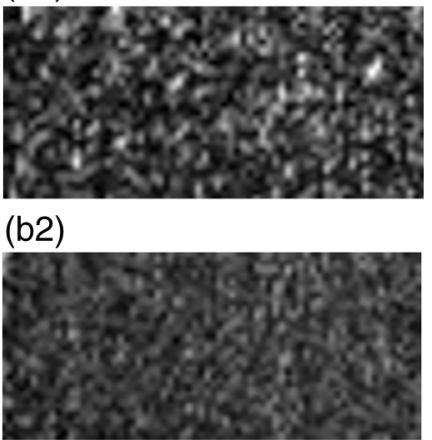

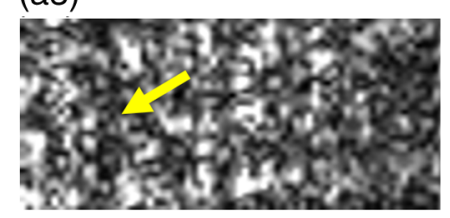

(b3)

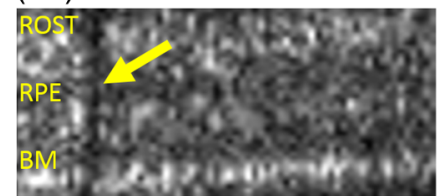

Fig. 5 Speckle-reduction test in human retina using modulated raster scan. (a) Reference raster B-scan image. Three ROls from NFL, GCL, and ORL are highlighted by (a1), (a2), and (a3), respectively. The size of each $\mathrm{ROI}$ is $430 \mu \mathrm{m}$ (lateral) $\times 23 \mu \mathrm{m}$ (axial). (b) The corresponding srB-scan image. The same three ROls are highlighted by (b1), (b2), and (b3), respectively. (a1)-(a3) The magnified views of the three highlighted ROIs in panel (a). (b1)-(b3) The magnified views of the three highlighted ROIs in panel (b). CNR and ENL values are calculated from all the selected ROls. The bottom three anatomical layers ROST, RPE, and BM are highlighted in panel (b3). The arrows in (a3) and (b3) highlight the same blood vessel shadow. All images are plotted with identical color bar.
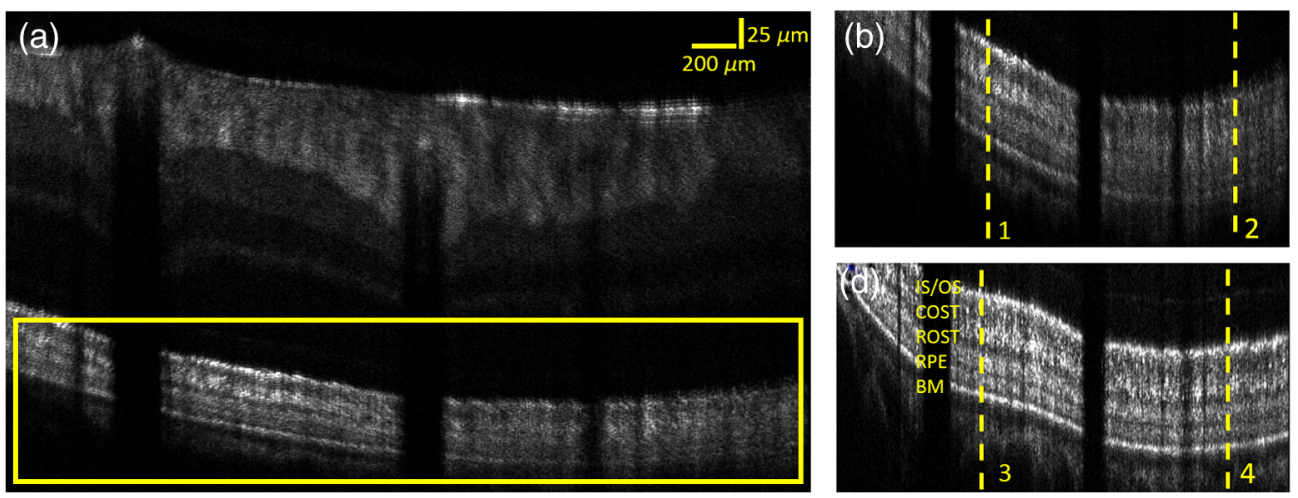

(c)
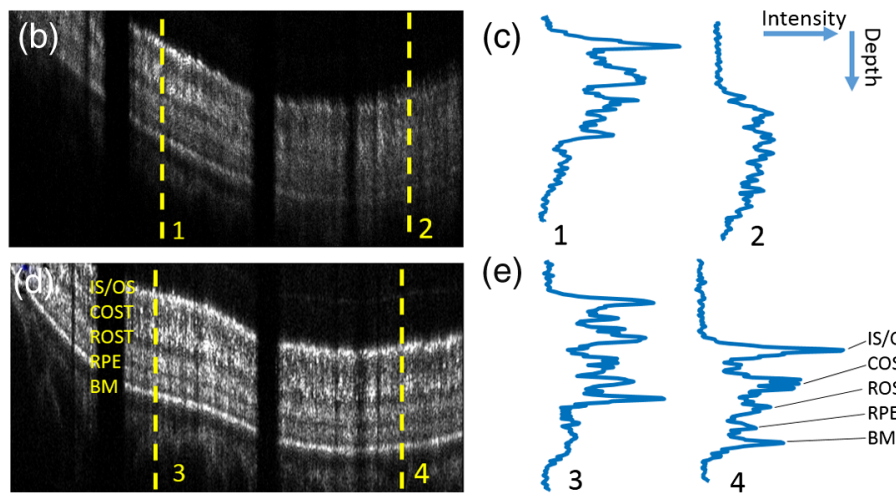

(e)
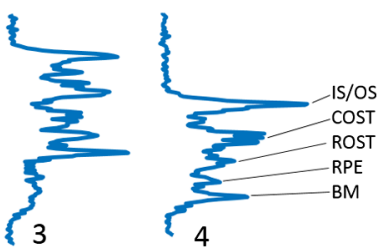

Fig. 6 Directly comparing averaged B-scan with srB-scan images from human retina. (a) Image scan from the same location as shown in Fig. 5(b) after averaging eight B-scans. (b) Magnified view of the outer retina region as highlighted in panel (a). (c) Two A-lines from the positions highlighted by 1 and 2 in panel (b). (d) Magnified view of the same outer retina region from the srB-scan shown in Fig. 5(b). Five anatomical layers are labeled. (e) Two A-lines from the positions highlighted by 3 and 4 in panel (d). All plotted A-lines are averaged three times laterally to reduce variation. All images are plotted on the same contrast scale as used in Fig. 5.

Table 2 compares the CNR and ENL values from the selected ROIs in the human retina for both raster and circular scans. In each instance, we see increased metric values in the srB-scan ROIs. Raster scans show an improvement in CNR of $2.25,2.00$, and $1.86 \mathrm{~dB}$, in the NFL, GCL, and ORL, respectively. Corresponding ENL improvements are 2.87, 2.72, and 2.52 times, respectively. Circular scans show an improvement in CNR of 2.08, 1.92, and $1.64 \mathrm{~dB}$, in the NFL, GCL, and ORL, respectively. Here, corresponding ENL improvements are $2.77,2.11$, and 1.81 times, respectively. Again, we attribute the slightly lower metric value increases in the ORL to the unavoidable inclusion of image background in the ROI, artificially contributing low pixel intensities to $\mu_{i}$ in the metrics. 
(a)
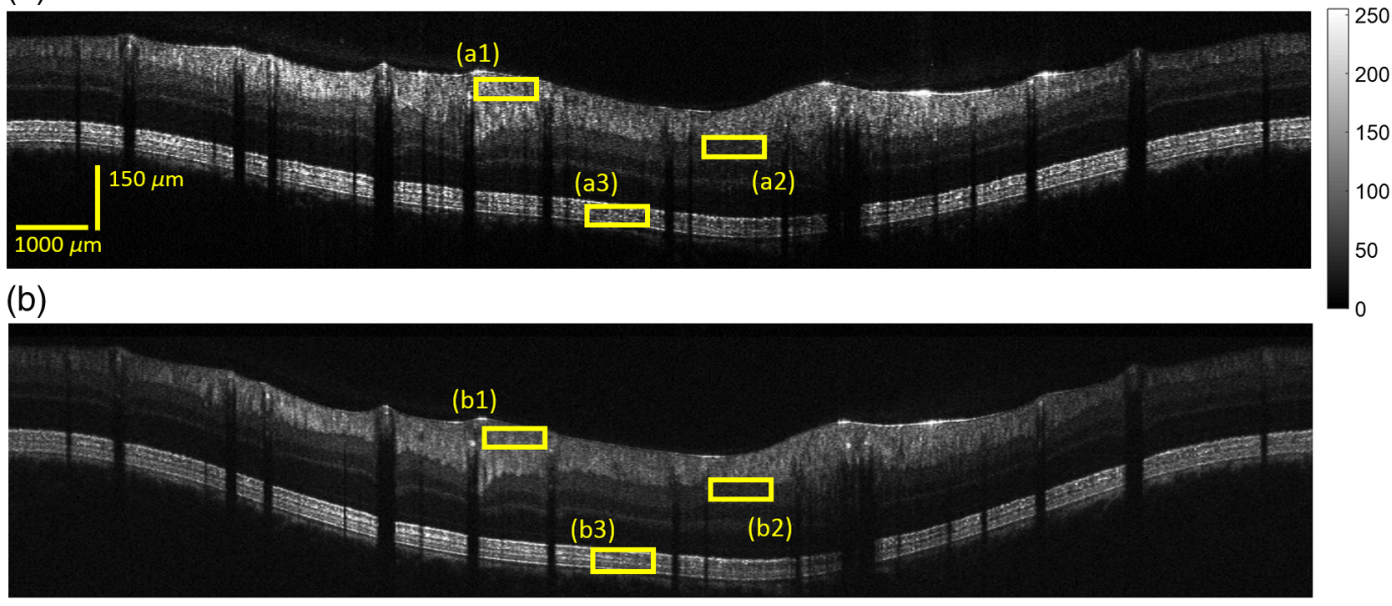

(a1)

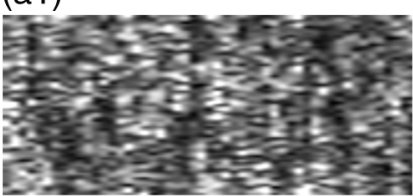

(b1)

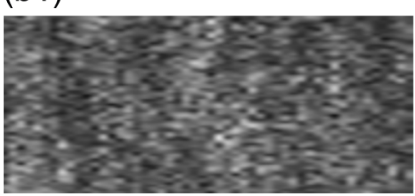

(a2)

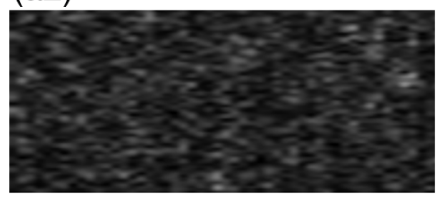

(b2)

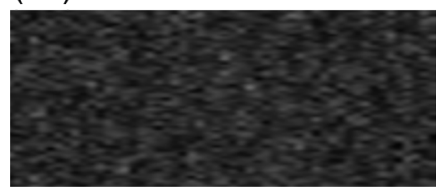

(a3)

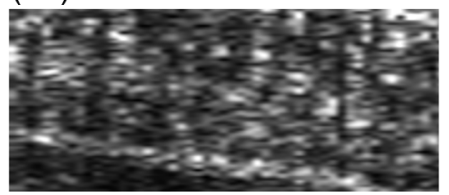

(b3)

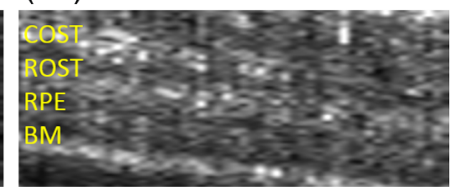

Fig. 7 Speckle-reduction test in human retina using modulated circular scan. (a) Reference circular Bscan image. Three ROIs from NFL, GCL, and ORL are highlighted by (a1), (a2), and (a3), respectively. The size of each ROI is $900 \mu \mathrm{m}$ (lateral) $\times 40 \mu \mathrm{m}$ (axial). (b) The corresponding srB-scan image. The same three ROIs are highlighted by (b1), (b2), and (b3), respectively. (a1)-(a3) The magnified views of the three highlighted ROls in panel (a). (b1)-(b3) The magnified views of the three highlighted ROIs in panel (b). CNR and ENL values are calculated from all the selected ROIs. The bottom four anatomical layers cone outer segment tips (COST), ROST, RPE, and BM are highlighted in panel (b3). All images are plotted with identical color bar.

Table 2 Image quality metric values from the ROls in the human retina from Figs. 5 and 7.

\begin{tabular}{lccccc}
\hline & & & & & \\
Scan type & ROI & $\begin{array}{c}\text { CNR (dB) } \\
\text { ref. B-scan }\end{array}$ & $\begin{array}{c}\text { CNR (db) } \\
\text { srB-scan }\end{array}$ & $\begin{array}{c}\text { ENL } \\
\text { ref. B-scan }\end{array}$ & $\begin{array}{c}\text { ENL } \\
\text { srB-scan }\end{array}$ \\
\hline Raster & NFL & 2.39 & 4.64 & 4.19 & 12.01 \\
Raster & GCL & 2.31 & 4.31 & 5.92 & 16.12 \\
Raster & ORL & 1.90 & 3.76 & 3.76 & 9.47 \\
Circular & NFL & 2.85 & 4.93 & 6.15 & 17.03 \\
Circular & GCL & 1.99 & 3.91 & 10.50 & 22.13 \\
Circular & ORL & 1.76 & 3.40 & 4.52 & 8.20 \\
\hline
\end{tabular}

\subsection{Speckle Reduction Test in Clinical Environment}

A clinical photographer without technical knowledge of the scanning protocol independently verified speckle reduction in the human retina using a commercial vis-OCT system. The photographer acquired images using the same procedure as acquiring normal raster scan images and received no additional training. Quality improvement in the clinical speckle-reduced images is comparable to the lab tests (Sec. 3.3). Figures 8(a) and $8(\mathrm{~b})$, respectively, show a reference B-scan and an srB-scan of the macula from a 37-year-old female volunteer. In the reference B-scan image, speckle particularly distorts the ORL, as shown in Fig. 8(c), preventing the delineation of fine anatomical structures, such as the RPE and BM. As a comparison, all five ORLs, including the RPE and BM, are clearly resolved in the magnified srB-scan image, as shown in Fig. 8(d). Quantitatively, the improvements in CNR and ENL from similar ROIs are comparable with what we achieved in lab tests.

\section{Discussion}

This study implemented, calibrated, and tested a scanning modulation technique for speckle reduction in vis-OCT. We addressed unique engineering constraints of clinical vis-OCT, including slower image acquisition speed, intrinsically reduced SNR, and the need to preserve structurally localized, highdetailed retinal information. As a comparison in near-infrared (NIR) OCT, good SNR can be achieved at higher imaging speeds, ${ }^{3}$ direct B-scan averaging is more feasible, and lower resolutions are less affected by image blurring. In our human imaging tests, where motions were high, direct B-scan averaging in vis-OCT proved unreliable for producing high-quality images at 
(a)

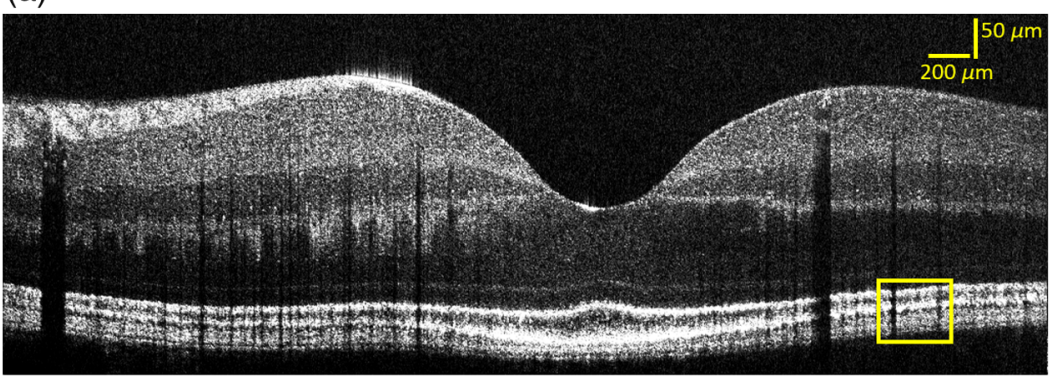

(b)

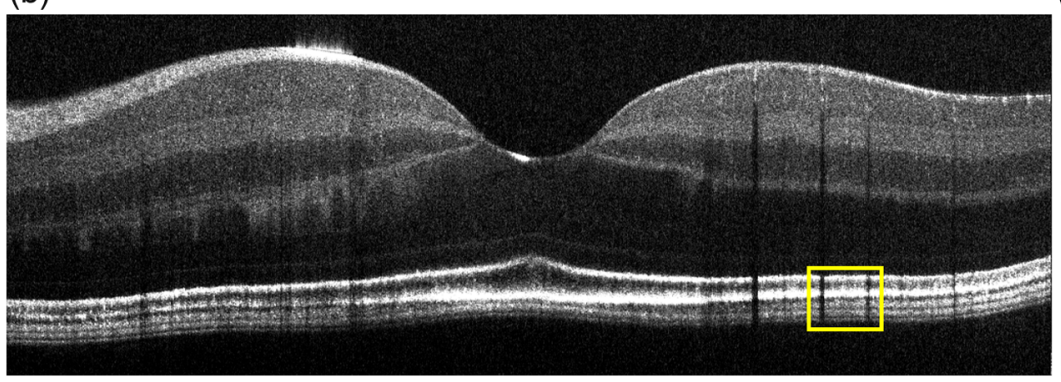

(c)

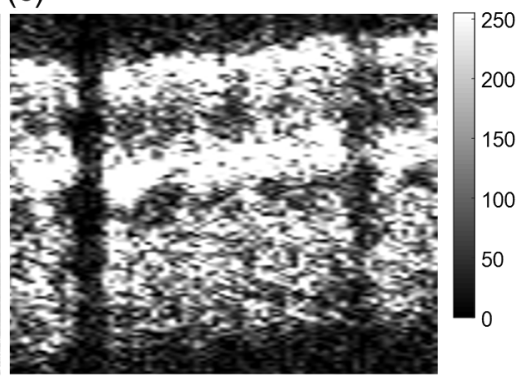

(d)

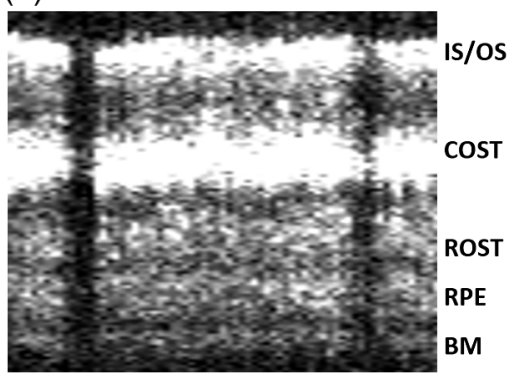

Fig. 8 Speckle-reduction test in human retina in clinical environment using modulated raster scan. (a) Reference raster B-scan image; (b) the corresponding srB-scan image; (c) magnified view of the region highlighted in panel (a); and (d) magnified view of the region highlighted in panel (b). The five anatomical layers, IS/OS, COST, ROST, RPE, and BM, are labeled. All images are plotted with identical color bar.

an A-line rate of $25 \mathrm{kHz}$, as shown in Figure 6. Our rectangular modulation of the scanning beam reliably produced specklereduced images in both mouse and human retinas without image blurring.

The ultimate target of vis-OCT retinal imaging is the clinical adoption, where increased resolution and spectroscopic analysis can improve the management of a variety of retinal diseases. ${ }^{16}$ Therefore, a primary design constraint of our speckle-reduction technique was the usability by photographers in the clinical environment, where advanced vis-OCT engineering skills are unavailable. It is unreasonable to expect a clinical photographer to make optical adjustments to the system or troubleshoot technical issues that arise. We implemented our speckle reduction in a clinical vis-OCT system at NYU Langone Medical Center simply via a software update. No additional optical calibrations were made to the clinical system, and no changes were made to the photographers' imaging protocol. Critically, the photographers achieved comparable image quality (Fig. 8) to experts in a controlled lab environment and the clinical images showed delineation of RPE and BM [Figs. 8(b) and 8(d)]. Micrometerscale basal linear and basal laminar deposits between RPE and $\mathrm{BM}$ are thought to be early indicators of macular degeneration, a leading cause of blindness. ${ }^{24} \mathrm{~A}$ future study in which these morphological changes are observed in vivo in the clinic may open a new window for the diagnosis and management of macular degeneration. Such capabilities have been previously unavailable in clinical NIR OCT systems due to reduced axial resolution. Presently, NIR OCT is limited to imaging larger scale drusen, ${ }^{24}$ which are developed only at a more advanced stage of macular degeneration.

Szkulmowski et al. ${ }^{10}$ performed averaging of offset A-lines in NIR OCT using a resonant scanner. One hardware challenge expressed in this study was fringe washout. In addition to the complication of adding hardware to the system, the resonant scanner continuously moves during a single camera exposure.
The axial component of the scanner velocity induces Doppler shifts to the interference fringe, thereby reducing the image SNR. ${ }^{26}$ To overcome this challenge, Szkulmowski et al. suggested minimizing camera exposure time (demonstrated at $5 \mu \mathrm{s}$ ) to reduce the effects of fringe washout. Such reduction of camera exposure time, however, would severely compromise image quality in vis-OCT, which typically requires camera exposures of $\sim 40 \mu$ s to achieve sufficiently high SNR in the human retina. Our scanning technique does not introduce additional fringe washout, as the scanning beam moves in discrete steps and is stationary during each camera exposure. Szkulmowski et al. experienced $\sim 6$-dB loss to peak signal-to-noise-ratio (PSNR) ${ }^{10}$ in scan-optimized images at an exposure time of $20 \mu \mathrm{s}$, which they attribute to fringe washout. We did not observe PSNR drop in our scan-optimized images at an exposure time of $40 \mu \mathrm{s}$. Although our discrete scanning trajectory is optimized for visOCT, it is expected to provide superior image quality in other OCT systems with higher exposure times when using the same sampling parameters as a resonance mirror-based technique.

Our scanning technique also addresses the trajectory limits of a single resonant mirror shown in Szkulmowski et al. Using discrete, software-controlled XY galvanometer scanners, we enabled multiple modulation waveforms across multiple coordinate systems (e.g., Cartesian and polar). There is promise for applying circular scan modulation for circumpapillary retinal oximetry, where speckles in blood may disrupt true spectroscopic signal. The velocity of blood (e.g., $\sim 0.014 \mu \mathrm{m} / \mu$ s for a $100-\mu \mathrm{m}$ diameter human retinal artery ${ }^{27}$ ) is not high enough to completely uncorrelate the local scattering structure of erythrocytes during consecutive A-lines (period of $40 \mu \mathrm{s}$ ). Therefore, A-line averaging across a regular circular scan is not perfectly efficient for reducing the effects of speckle. By scanning a larger volume, our modulated circular scan is expected to acquire more uncorrelated speckle patterns in blood using the same number of A-lines as a regular circular scan. Furthermore, the modulated 
scan pattern is not expected to disrupt the SNR of the blood signal since it does not induce additional fringe washout.

Next, by selecting a rectangular waveform, we best preserved the lateral resolution along the B-scan axis and took full advantage of the space available along the orthogonal axis for speckle pattern decorrelation. In comparison, a resonant scanner can only oscillate along a single axis. Furthermore, the reported resonant scanner uses a sinusoidal waveform, in which each mirror deflection along the orthogonal axis also carries a component along the B-scan axis. In this case, averaging consecutive A-lines will reduce the lateral resolution along the B-scan axis. In addition, the sinusoidal trajectory makes orthogonal step size, $d$, nonlinear across each scan of the orthogonal axis. Our findings suggest that image quality is optimized when $d$ is approximately equal to the focused spot diameter along the orthogonal axis. Precise optimization is not possible with a nonlinear trajectory. Szkulmowski et al. partially overcame this by only acquiring A-lines along the pseudolinear region of every other sinusoidal edge, wasting a portion of light exposure on the retina. However, this is still less efficient than scanning with a rectangular wave, in which all spaces along the orthogonal axis are linear and can be fully utilized.

Acquiring A-lines along the orthogonal axis increases imaging time by a factor of $n=8$. This limits the total number of srB-scans per acquisition to 16 for an imaging time of $\sim 5 \mathrm{~s}$. Naturally, 16 raster srB-scans are not feasible to reconstruct a high-quality en-face image of the human retina. A potential solution is to decompose each srB-scan with $n=8$ into eight regular B-scans orthogonal axis, each of 1024 A-lines. If the spacing between each srB-scan is $d$, then we can decompose $16 \mathrm{srB}$ scans into 128 equidistant regular B-scans separated by the distance $d$. By reducing the number of srA-lines per srB-scan to 4096 at $n=8$, we can decompose the full raster scan into 512 A-lines $-256 \mathrm{~B}$-scans, and so on. The user may view regular B-scans or srB-scans at a location of interest in the en-face image. Rather than choosing between a high-quality en-face image and high-quality B-scans, the user may adjust scanning parameters to acquire both simultaneously. We recognize, however, that the aforementioned solution is still not as optimal as a regularly raster-scanned en-face image due to motion and that extra image registrations may be required.

\section{Conclusion}

We showed that our orthogonal modulation protocol significantly reduced the speckle for both raster and circular scans in vis-OCT without additional hardware. This protocol was robust against retinal motions. Furthermore, we showed that speckle-reduced vis-OCT imaging does not require additional training for a clinical photographer to operate, allowing smooth clinical translation. We designed this orthogonal modulation protocol to be compatible with arbitrary scanning trajectories and demonstrated such capability in circular scans for the first time.

In the mouse retinas, we showed that up to a $2.35-\mathrm{dB}(116 \%)$ improvement in CNR and 3.1-fold increase in ENL can be achieved. We recognized that an anesthetized and stabilized mouse retina had negligible motion, allowing for simple B-scan averaging, and further conducted human retinal imaging. Consistently improved image qualities after speckle reduction were shown in human retinas using both an experimental and a clinical vis-OCT system and provide comparable results. Here, we showed that up to a $2.25-\mathrm{dB}$ (94\%) improvement in
CNR and 2.87-fold increase in ENL can be achieved. We also showed that reducing speckle via direct B-scan averaging is not as reliable for preserving the fine features, due to retinal motion. Our speckle-reduction method offered a local sampling rate of $3125 \mathrm{~Hz}$ (Sec. 2.1), which is well beyond the motion frequency.

In the future, we will further improve the speckle-reduction performance, especially in clinics, by providing real-time feedbacks of CNR and ENL so that the photographer can adjust imaging parameters to achieve optimal image quality for different eye conditions. In addition to exploring clinical benefits of discriminating minute anatomical features, such as the RPE and $\mathrm{BM}$, we will also investigate whether circular scan modulation can improve the accuracy of measuring retinal oxygen saturation because an srB-scan samples a larger retinal volume than a regular B-scan image.

\section{Disclosures}

R.K., Y.W., J.S.S., and H.F.Z. have financial interests in Opticent Health, which did not support this work. Other authors declare no conflicts of interest related to this article.

\section{Acknowledgments}

This work was supported in part by NIH Grants Nos. R01EY026078, DP3DK108248, R01EY029121, R01EY028304, R44EY026466, and T32EY25202. L.B. was supported by the NSF Graduate Research Fellowship 1000260620. The authors would like to thank David Miller and Rozita Ghassabi for their helpful discussions.

\section{References}

1. D. Huang et al., "Optical coherence tomography," Science 254, 1178-1181 (1991).

2. M. R. Hee et al., "Optical coherence tomography of the human retina," Arch. Ophthalmol. 113, 325-332 (1995).

3. M. Wojtkowski, "High-speed optical coherence tomography: basics and applications," Appl. Opt. 49, D30-D61 (2010).

4. J. M. Schmitt, S. H. Xiang, and K. M. Yung, "Speckle in optical coherence tomography," J. Biomed. Opt. 4(1), 95-105 (1999).

5. M. R. Hee et al., "Optical coherence tomography of age-related macular degeneration and choroidal neovascularization," Ophthalmology 103, 1260-1270 (1996).

6. M. R. Hee et al., "Optical coherence tomography of central serous chorioretinopathy," Am. J. Ophthalmol. 120, 65-74 (1995).

7. A. Ozcan et al., "Speckle reduction in optical coherence tomography images using digital filtering," J. Opt. Soc. Am. A 24, 1901-1910 (2007).

8. M. Bashkansky and J. Reintjes, "Statistics and reduction of speckle in optical coherence tomography," Opt. Lett. 25, 545-547 (2000).

9. A. Sakamoto, M. Hangai, and N. Yoshimura, "Spectral-domain optical coherence tomography with multiple B-scan averaging for enhanced imaging of retinal diseases," Ophthalmology 115, 1071-1078 (2008).

10. M. Szkulmowski et al., "Efficient reduction of speckle noise in optical coherence tomography," Opt. Express 20, 1337-1359 (2012).

11. A. E. Desjardins et al., "Speckle reduction in OCT using massivelyparallel detection and frequency-domain ranging," Opt. Express 14, 4736-4745 (2006).

12. M. Pircher et al., "Speckle reduction in optical coherence tomography by frequency compounding," J. Biomed. Opt. 8(3), 565-569 (2003).

13. Y. Zhao et al., "Real-time speckle reduction in optical coherence tomography using the dual window method," Biomed. Opt. Express 9, 616-622 (2018)

14. O. Liba et al., "Speckle-modulating optical coherence tomography in living mice and humans," Nat. Commun. 8, 15845 (2017).

15. C. Cuartas-Velez et al., "Volumetric non-local-means based speckle reduction for optical coherence tomography," Biomed. Opt. Express 9, 3354-3372 (2018). 
16. X. Shu, L. Beckmann, and H. F. Zhang, "Visible-light optical coherence tomography: a review," J. Biomed. Opt. 22(12), 121707 (2017).

17. J. Yi et al., "Visible-light optical coherence tomography for retinal oximetry," Opt. Lett. 38, 1796-1798 (2013).

18. J. Yi et al., "Human retinal imaging using visible-light optical coherence tomography guided by scanning laser ophthalmoscopy," Biomed. Opt. Express 6, 3701-3713 (2015).

19. S. P. Chong et al., "Ultrahigh resolution retinal imaging by visible light OCT with longitudinal achromatization," Biomed. Opt. Express 9, 1477-1491 (2018).

20. S. Martinez-Conde, S. L. Macknik, and D. H. Hubel, "The role of fixational eye movements in visual perception," Nat. Rev. Neurosci. 5, 229-240 (2004).

21. D. C. Adler, T. H. Ko, and J. G. Fujimoto, "Speckle reduction in optical coherence tomography images by use of a spatially adaptive wavelet filter," Opt. Lett. 29, 2878-2880 (2004)

22. B. T. Soetikno et al., "Visible-light optical coherence tomography oximetry based on circumpapillary scan and graph-search segmentation," Biomed. Opt. Express 9, 3640-3652 (2018).

23. J. C. Booij et al., "The dynamic nature of Bruch's membrane," Prog. Retinal Eye Res. 29, 1-18 (2010).

24. C. A. Curcio et al., "The oil spill in ageing Bruch membrane," Br. J. Ophthalmol. 95, 1638-1645 (2011).

25. M. Guizar-Sicairos, S. T. Thurman, and J. R. Fienup, "Efficient subpixel image registration algorithms," Opt. Lett. 33, 156-158 (2008).

26. S. H. Yun et al., "Motion artifacts in optical coherence tomography with frequency-domain ranging," Opt. Express 12, 2977-2998 (2004).

27. C. E. Riva et al., "Blood velocity and volumetric flow-rate in human retinal-vessels," Invest. Ophthalmol. Visual Sci. 26, 1124-1132 (1985).

lan Rubinoff is a second-year $\mathrm{PhD}$ student in biomedical engineering at the Functional Optical Imaging Lab (FOIL) at Northwestern University. In 2017, he graduated from Lehigh University with a BS degree in electrical engineering and physics. His research interests include innovating visible-light optical coherence tomography (vis-OCT) technology, optics, and signal processing.

Lisa Beckmann is a third-year PhD student in biomedical engineering at the FOIL at Northwestern University. In 2016, she graduated from the California Institute of Technology in biomedical engineering. Her research interests include using vis-OCT to study eye pathologies, optics, and animal model imaging.

Yuanbo Wang is a senior project engineer at Opticent Health in Evanston, Illinois. He graduated with a $\mathrm{PhD}$ in biomedical engineering from the University of Missouri-Columbia, where he studied polarization sensitive OCT and fiber tractography.

Amani A. Fawzi is the Cyrus Tang and Lee Jampol Professor of Ophthalmology at the Feinberg School of Medicine, Northwestern
University, and a practicing ophthalmologist at Northwestern Memorial Hospital. She previously worked at the Jules Stein Eye Institute at UCLA and the Doheny Retina Institute at USC. Her research interests include translational approaches to age-related macular degeneration and ischemic retinal diseases.

Xiaorong Liu is an assistant professor of biology and psychology at the University of Virginia. She received her PhD from the University of Virginia in 2002 and received postdoctoral training at the Scripps Research Institute and the University of California, San Francisco. Her research interests include the structural and functional development of retinal ganglion cells and how they degenerate in glaucoma.

Jenna Tauber is an MD candidate at the New York University (NYU) School of Medicine. She received her BS degree in communications from Cornell University.

Katie Jones is a clinical photographer and lab supervisor at the Advanced Ophthalmic Imaging Laboratory, NYU. She graduated in 2013 with a BS degree in biology from the University of Pittsburgh.

Hiroshi Ishikawa is a professor in the Department of Ophthalmology, NYU and administrative director of the Ophthalmic Imaging Center. He received his MD from Mie University in Japan in 1989.

Joel S. Schuman is a professor and chairman of the Department of Ophthalmology, NYU. He received his MD from Mt. Sinai School of Medicine in 1984 and served as professor and chairman of the Department of Ophthalmology at the University of Pittsburgh from 2003 to 2016 . He is a coinventor of OCT and a pioneer in the study and treatment of glaucoma.

Roman Kuranov is the head of product engineering and development at Opticent Health in Evanston, Illinois, and a research specialist at Northwestern University. He received his PhD in laser physics from the Institute of Applied Physics of the Russian Academy of Sciences and did his postdoctoral training at the University of Texas, Galveston. He previously worked as principal scientist in Wasatch Photonics and as an instructor and researcher at the University of Texas, San Antonio.

Hao F. Zhang is a professor in the Department of Biomedical Engineering at Northwestern University. He leads the FOIL, which seeks to develop and apply new optical imaging technologies for biological study and clinical use. His lab pioneered the development of vis-OCT for high-resolution and functional retinal imaging. He received his $\mathrm{PhD}$ in biomedical engineering from the Texas $\mathrm{A} \& \mathrm{M}$ University in 2006 and received his postdoctoral training from the Department of Biomedical Engineering at Washington University in St. Louis. Zhang also cofounded Opticent Health, which seeks to translate vis-OCT to the clinic. 